\title{
Experimental Evidence of Coherence Resonance in a Time-Delayed Bistable System
}

\author{
Mikel Arizaleta Arteaga, ${ }^{1,2}, *$ Miguel Valencia, ${ }^{2}$ Marc Sciamanna, ${ }^{3}$ Hugo Thienpont, ${ }^{2}$ \\ Manuel López-Amo, ${ }^{1}$ and Krassimir Panajotov ${ }^{2, \dagger}$ \\ ${ }^{1}$ Department of Electrical and Electronic Engineering, Public University of Navarra, 31006 Pamplona, Spain \\ ${ }^{2}$ Department of Applied Physics and Photonics (IR-TONA), Vrije Universiteit Brussel (VUB), B-1050 Brussels, Belgium \\ ${ }^{3}$ Supélec, LMOPS CNRS UMR-7132, 57070 Metz, France
}

(Received 19 January 2007; published 13 July 2007)

\begin{abstract}
We report on the experimental observation of coherence resonance in a bistable system with delay. Our system consists of a vertical-cavity surface-emitting laser subject to time-delayed optical feedback simultaneously from a long and from an extremely short external cavity. Coherence resonance is experimentally proven by analysis of the residence time distribution of the polarization mode-hopping regime and of the signal to noise ratio in the power spectrum.
\end{abstract}

DOI: 10.1103/PhysRevLett.99.023903

PACS numbers: 42.65.Sf, 05.40.Ca, 42.55.Px

It is commonly accepted now that noise can play a constructive role in nonlinear dynamical systems. After the seminal paper of Benzi, Sutera, and Vulpiani [1], the phenomenon of stochastic resonance, namely, the fact that adding noise can better synchronize a dynamical system to an external periodic signal, has attracted a lot of interest in biology [2], physics [3], chemistry [4], etc. (for a review, see, e.g., [5]). It has recently been realized that noise can enhance regular dynamics in nonlinear systems even in the absence of an external signal, when an internal time scale is present in the system [6]. This phenomenon was initially considered as stochastic resonance in an autonomous system and later named coherence resonance (CR) [7]. CR was first predicted for excitable dynamical systems [6-8], i.e., systems that emit quasiregular pulses as a result of an excitation threshold and with a refractory period. It has been demonstrated experimentally in excitable electronic circuits [9], semiconductor lasers subject to optical feedback and driven into chaotic excitable dynamics [10], and Belousov-Zhabotinsky chemical reaction systems [11]. For a recent review, see Ref. [12]. CR has also been predicted as a noisy precursor of limit cycle bifurcations in nonlinear systems [8]. This mechanism for CR has been verified experimentally in electrochemical systems [13] and close to Hopf bifurcations in multisection semiconductor lasers [14]. Theoretical works on different models furthermore reported that not only excitable but also bistable or multistable systems driven by noise can exhibit CR $[15,16]$, as also confirmed experimentally in bistable chaotic electronic Chua circuits [17]. Recently, CR has been predicted in another class of systems, which exhibit bistability together with time delay [18]. These systems are interesting, considering also that delay is an inherent property of Nature, with examples found in biology [19], economics [20], and chemistry [21].

In this Letter, we present experimental evidence of CR in an optical bistable time-delayed system, namely, a vertical-cavity surface-emitting laser (VCSEL) subject to optical feedback. VCSELs are known to exhibit bistability between two nearly degenerate linearly polarized (LP) fundamental transverse modes [22]. This has allowed for the experimental observation of stochastic resonance in polarization mode hopping induced by noise [23]. In the presence of time-delayed optical feedback, the polarization mode hopping is accompanied by anticorrelated oscillations at the external-cavity frequency in LP mode intensities [24]. These dynamics strongly enhance the probability for polarization switchings at the delay time and multiples of it. As a result, the residence time distribution (RTD) of the LP modes is strongly modified, showing peaks at the delay time and multiples of it [25]. These dynamics can result in $\mathrm{CR}$, as predicted numerically in Ref. [25] and as confirmed here experimentally.

Our experimental setup is described in Fig. 1. A VCSEL is subject to optical feedback from two external cavities. The first cavity, formed between the VCSEL output mirror and a fiber facet with enhanced reflectivity, is an extremely short external cavity (ESEC) and is used to tune the VCSEL polarization-switching current [26]. The second cavity is the optical fiber itself and introduces a time delay of $\tau \approx 27 \mathrm{~ns}\left(L_{2} \approx 2.7 \mathrm{~m}\right)$ in the system. We use an oxideconfined GaAs/InGaAs quantum well VCSEL, emitting at

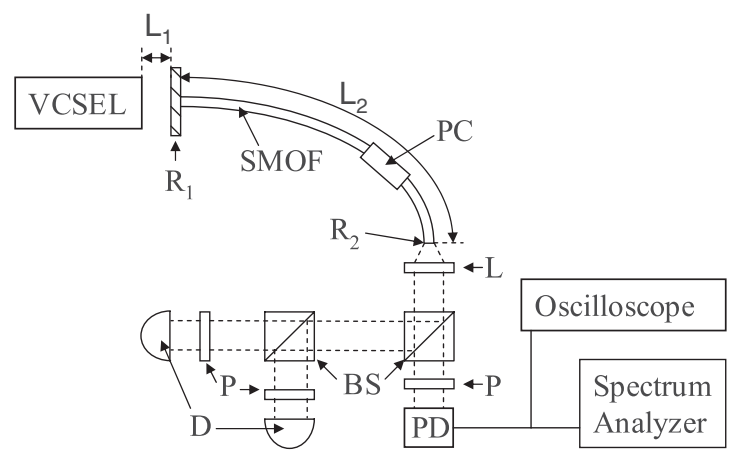

FIG. 1. Experimental setup. SMOF, single-mode optical fiber; PC, polarization controller; L, lens; P, polarizer; BS, beam splitter; D, photodiode; PD, high speed photodiode. 
$1.005 \mu \mathrm{m}$ in its fundamental transverse mode up to 1.75 times the threshold current [26]. In order to create the ESEC, we place a single-mode optical fiber at a few micrometers from the VCSEL. The optical feedback from the ESEC is enhanced by gluing a mirror of $R_{1}=23 \%$ reflectivity to the fiber facet. $L_{1}$ is changed by moving the VCSEL by means of one of the three piezo components of the stage on which it is mounted in steps of $20 \mathrm{~nm}$ and with a precision of $5 \mathrm{~nm}$. A CCD camera (with resolution $1 \mu \mathrm{m}$ ) is used to measure the absolute value of $L_{1}$. The values of $L_{1}$ reported in the following are relative-the zero corresponds to $L_{1}$ smaller than $20 \mu \mathrm{m}$ [26]. The light emitted by the VCSEL is butt-coupled into a $2.7 \mathrm{~m}$ long singlemode fiber through $R_{1}$ (see Fig. 1). A FPC-100 polarization controller is used in order to compensate any possible unintentional birefringence introduced in the fiber. The far facet of the fiber is either ended in an angled polished fiber-optic connector to have no feedback from the long external cavity $\left(R_{2}=0\right)$ or perpendicularly cleaved providing a reflectivity of $4 \%\left(R_{2}=4 \%\right)$. The light coming out from the fiber allows us to measure the polarizationresolved output intensity. To obtain the time traces, a photodiode of $12 \mathrm{GHz}$ bandwidth and a oscilloscope of $2.4 \mathrm{GHz}$ bandwidth are used. The power spectrum is simultaneously measured using a $30 \mathrm{GHz}$ bandwidth electrical spectrum analyzer.

The above described experimental setup allows, by nanometric tuning of the ESEC length $L_{1}$, a precise control of the current at which polarization switching (PS) between the two linearly polarized fundamental transverse modes of the VCSEL occurs [26]. This allows us the same flexibility as in the numerical prediction of CR in Ref. [25], where the PS current is selected by modifying the net gain difference between the LP modes. Figure 2(a) shows the PS current when increasing (solid black line) and decreasing (gray line) the injection current as a function of the ESEC length $\left(L_{1}\right)$. All of the experiments reported here are performed with $L_{1}=9.5 \mu \mathrm{m}$. With such a value for $L_{1}$ [cut A in Fig. 2(a)] and in the absence of feedback from the

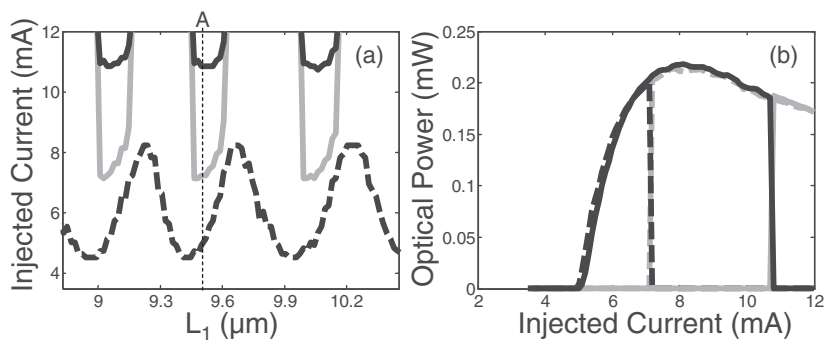

FIG. 2. (a) Polarization-switching current when increasing (solid black curve) and decreasing (gray curve) the injection current versus $L_{1}$. The black dashed line represents the threshold current. (b) Experimentally obtained polarization-resolved power versus current curve at $L_{1}=9.5 \mu \mathrm{m}$. Gray (black) line represents the low (high) frequency LP mode, while solid (dashed) lines represent the two LP modes for slowly increasing (decreasing) the injection current $(0.1 \mathrm{~mA} / \mathrm{s})$. long external cavity $\left(R_{2}=0\right)$, the polarization-resolved power versus current curve of the VCSEL shows PS between the two orthogonal linearly polarized fundamental transverse modes as shown in Fig. 2(b). When adding feedback from the long external cavity by perpendicularly cleaving the far end of the fiber $\left(R_{2}=4 \%\right)$, mode hopping between the two LP modes of the VCSEL occurs. In the presence of mode hopping, we define the LP mode asymmetry factor as $\Gamma=P_{L} / P_{H}$, where $P_{L}$ and $P_{H}$ are the time-averaged power of the low and the high frequency LP modes, respectively.

In the experiments, an external noise of zero mean value and a flat spectrum up to $300 \mathrm{MHz}$ is added to the injection current to observe the coherence of the polarizationresolved output power when varying the noise strength. Several ways of quantifying the coherence of the signal have been used in the literature. In the first experimental paper on stochastic resonance in an optical system, the maximum of the signal to noise ratio (SNR) of the peak at the forcing frequency was identified as a maximum of synchronization of the output signal [3]. Alternatively, the probability density of the residence times was suggested as an indicator to look for a resonance condition $[27,28]$. The experimental setup presented in Fig. 1 allows us to simultaneously check both indicators.

The residence times for the two LP modes are measured from the stored oscilloscope time trace of one LP mode alone by exploiting their anticorrelation behavior: The $\tau_{1}$ $\left(\tau_{2}\right)$ residence time is defined as the time spent above (below) a reference level (corresponding to one-half of the total unpolarized intensity) between two consecutive crossings of that level (see Fig. 3). Hereafter, we use the joint residence time $\left[\tau_{\text {JRT }}\right.$ in Fig. $\left.3(\mathrm{~b})\right]$, defined as the time interval needed for the system to visit the two LP modes consecutively [25]. The RTD of the two LP modes and the joint RTD (JRTD) are presented in Fig. 3(c). Because of large asymmetry $(\Gamma=8.5)$, the statistics of the high frequency LP mode shows a maximum at very short time scales, while the statistics of the low frequency mode shows a maximum just below $\tau$ (the small maximum on the left side is due to the noise added to the VCSEL current). The appearance of events for $t<\tau$ makes clear the difference of our optically delayed VCSEL system from the situation of optoelectronic feedback studied in Ref. [29]. In Ref. [29], the VCSEL subjected to a polarization selective optoelectronic feedback is driven into an excitable dynamics. As characteristic for excitable system, it cannot react during its refractory time equal to $\tau$ in that case, which gives no event for $t<\tau$ in the LP mode RTD. In our polarization insensitive all-optical feedback configuration, the system is bistable, allowing noise-induced switching also during the delay time $\tau$. The statistics of the JRTD shows a clear maximum at the delay time [25]. Furthermore, by using the JRTD we are able to observe the periodicity of the LP mode intensity irrespectively of the LP mode asymmetry, e.g., for $\Gamma>1$. Increasing the asymmetry factor $\Gamma$, the VCSEL stays more in one of the two LP 

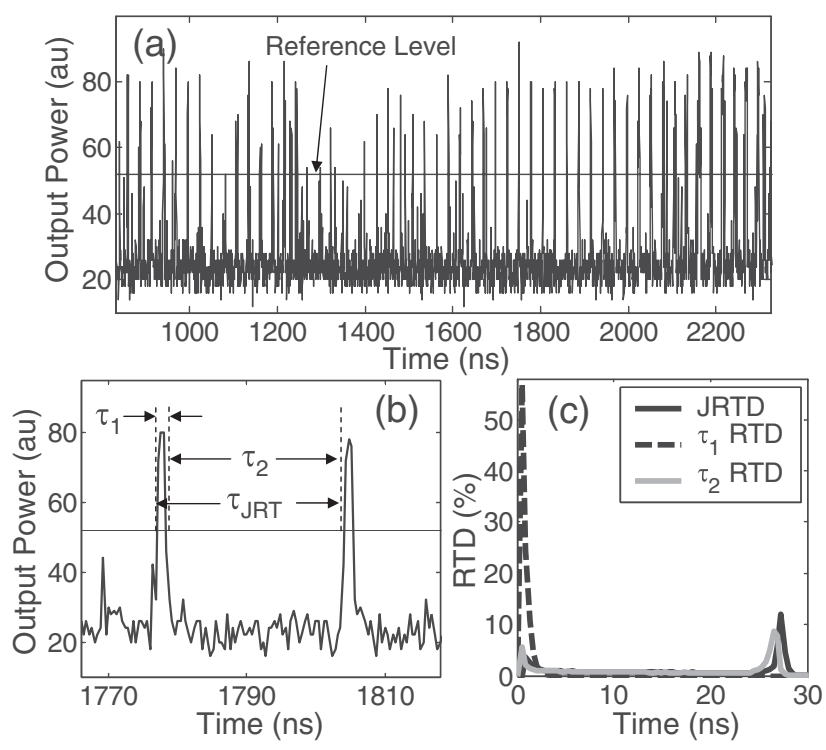

FIG. 3. (a) Time trace of polarization mode hopping (power emitted in the high frequency LP mode when $\Gamma=8.5$ ) influenced by time-delayed optical feedback $(\tau \simeq 27 \mathrm{~ns})$ with an enlargement in (b). (c) RTD of $\tau_{1}, \tau_{2}$, and $\tau_{\mathrm{JRT}}$.

modes (the low frequency one), and the picture of double potential wells for the two LP modes [30] becomes more and more asymmetric. The joint residence time distribution is governed by the increased part of the barrier between the two asymmetric potential wells.

Figure 4 shows the JRTD measured at different noise intensities; each one is calculated from 11 time traces of $4 \times 10^{6}$ points each. The time between measured samples is set to $400 \mathrm{ps}$. The VCSEL is biased at $9.05 \mathrm{~mA}(\Gamma=45)$. If the noise is weak, the system needs a lot of time to consecutively visit the two stable states, and the peak of the JRTD at the external-cavity round-trip time ( $\tau \approx 27 \mathrm{~ns})$ is very small [see Fig. 4(a)]. As the noise strength is increased, the peak at 27 ns dramatically increases, reaching

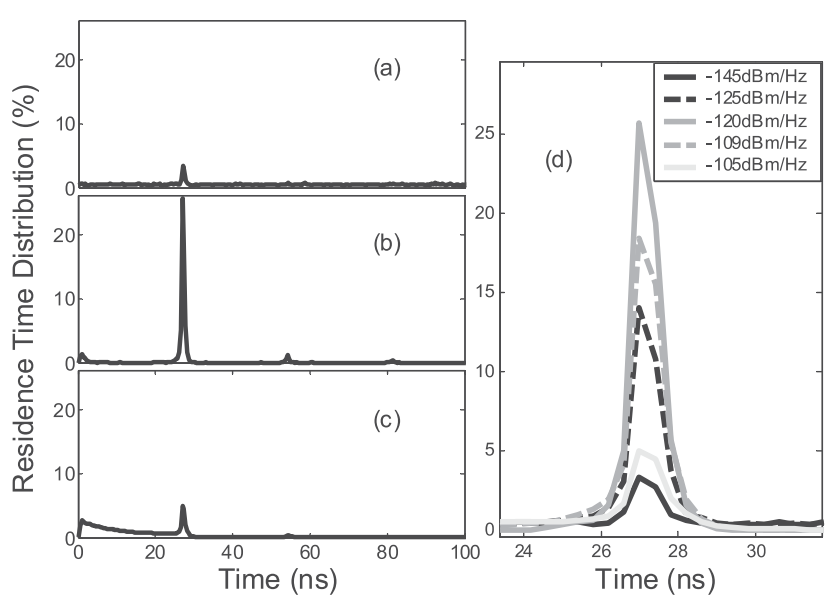

FIG. 4. Joint residence time distribution for external noise set to (a) -145 , (b) -120 , and (c) $-105 \mathrm{dBm} / \mathrm{Hz}$. (d) Zoom of the peak at 27 ns for different noise intensities. its maximum at $-120 \mathrm{dBm} / \mathrm{Hz}$ [see Figs. 4(b) and 4(d)]. For higher noise intensities, more and more fast polarization switchings occur, and the background masks the peak structure [see Fig. 4(c)].

The selection of a proper indicator for monitoring coherence resonance from RTDs is not trivial $[23,31]$. Here we use the area of the first peak of the JRTD once the background is subtracted. In order to do so, we numerically fit the JRTD with an exponential decay plus a Gaussian peak as in Ref. [23]. Figure 5 shows how the area of the first peak of the JRTD varies as the noise intensity is increased. The area of the peak is first increased and then decreased, showing a maximum for a noise intensity of $-120 \mathrm{dBm} / \mathrm{Hz}$. This constitutes clear evidence of coherence resonance in such a system.

Simultaneously with the time-trace acquisition, we measure the spectrum of the polarization-resolved output power with the electrical spectrum analyzer. Figure 6 shows the power spectra measured at three noise intensities. At a low noise strength, small peaks at the externalcavity frequency $(1 / \tau \approx 37 \mathrm{MHz})$ and its multiples can be observed in the spectrum. As the noise strength is increased, both the peak at $37 \mathrm{MHz}$ and the background noise level become higher [see Fig. 6(a)]. However, the signal to noise ratio of the peak first increases and then decreases as the noise intensity is increased, showing a maximum at $-107 \mathrm{dBm} / \mathrm{Hz}$ [see Fig. 6(b)]. This maximum occurs at a higher noise level than the maximum of the area of the first peak of the JRTD, in a similar way as reported in the case of stochastic resonance [5,30].

Now we decrease the injection current in such a way that $\Gamma=7.5$. The measured SNR and the area of the first peak of the JRTD versus noise intensity are presented in Fig. 7. Again, a maximum in the SNR versus injected noise strength is found [see Fig. 7(a)], which indicates coherence resonance but at a smaller noise intensity level. This result is consistent with the physical meaning of $\Gamma$, which relates to the distance of the bias current to the current at which mode-switching symmetry is obtained and, therefore, to the asymmetry of the potential wells and the barrier heights. A decrease of $\Gamma$ implies a decrease of the higher part of the asymmetric potential barrier between the two potential wells. On the other hand, the shift towards

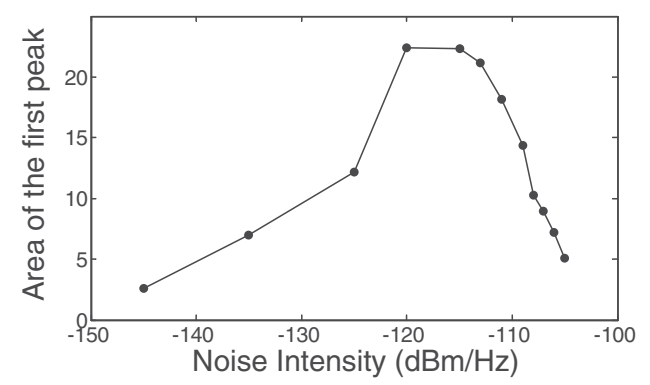

FIG. 5. Area of the first peak of the JRTD shown in Fig. 4 as a function of the input noise. The background has been subtracted before calculating the area and $\Gamma=45$. 


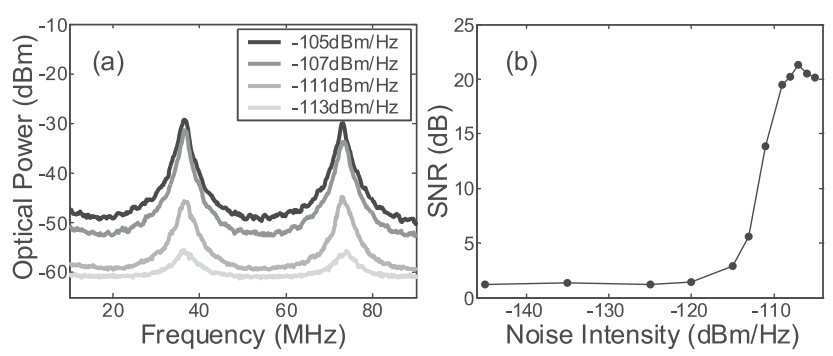

FIG. 6. (a) Electrical spectrum of the polarization-resolved output power at different noise intensities from -105 to $-145 \mathrm{dBm} / \mathrm{Hz}$. (b) Signal to noise ratio of the first peak in the electrical spectrum (at around $37 \mathrm{MHz}$ ). $\Gamma=45$.

smaller noise levels of the resonance condition moves the maximum of the area of the first peak of the JRTD out of the noise range of our generator, and now only a decreasing slope can be observed in the area versus the noise curve [see Fig. 7(b)]. The decreasing of the optimal noise intensity at which resonance is achieved when decreasing the LP mode asymmetry factor $\Gamma$ can be observed in Fig. 7(c).

In summary, we have combined here the VCSEL unique polarization properties with time-delayed optical feedback in order to experimentally confirm the general concept of coherence resonance in a bistable system with delay, as it was first predicted theoretically $[18,25]$. Coherence resonance is experimentally proven using two different criteria: the signal to noise ratio of the spectrum and the probability density of the joint residence time of the polarization mode

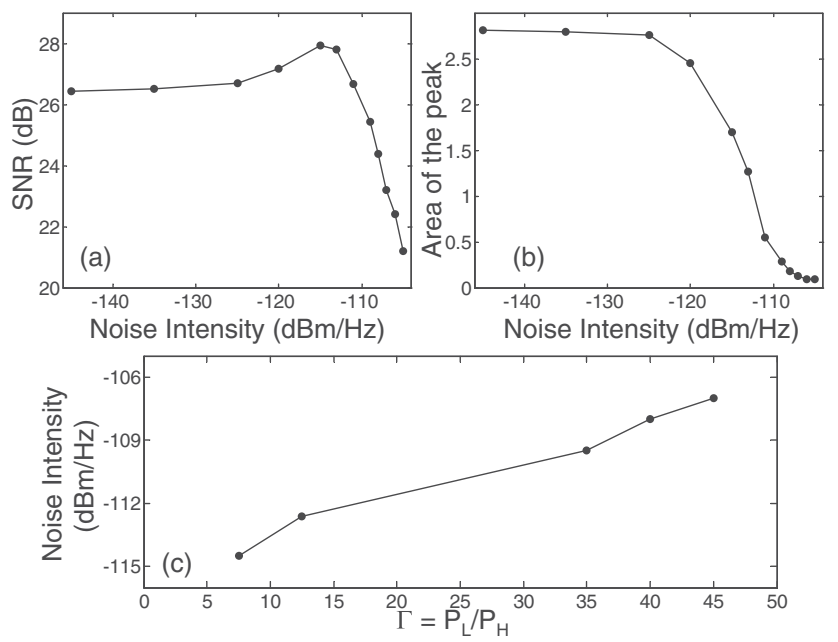

FIG. 7. (a) Signal to noise ratio of the first peak in the electrical spectrum (at around $37 \mathrm{MHz}$ ) as a function of the input noise. (b) Area of the first peak of the JRTD plotted as a function of the input noise. The background has been subtracted before the calculation of the area is made. In (a) and (b), $\Gamma=7.5$. (c) Intensity of injected noise at which the resonance condition according to the SNR indicator is achieved for different values of the LP mode asymmetry factor $\Gamma$. hopping. Experimentally, the noise at which the resonance condition occurs appears to be lower when using the JRTD as an indicator than when using the SNR. The resonance condition is experimentally found to be shifted towards lower noise levels as the LP mode asymmetry factor $\Gamma$ is decreased.

The authors acknowledge the financial support of the Public University of Navarra, IAP 6/10-Belgian Science Policy, FWO Flanders, GOA and OZR of the VUB, Conseil Régional de Lorraine, and Project No. TEC2004-05936-C02-01 of the MEC (Spain).

*mikel.arizaleta@unavarra.es

${ }^{\dagger}$ Also at Institute of Solid State Physics, 1784 Sofia, Bulgaria.

[1] R. Benzi, A. Sutera, and A. Vulpiani, J. Phys. A 14, L453 (1981).

[2] J. Douglass et al., Nature (London) 365, 337 (1993).

[3] B. McNamara, K. Wiesenfeld, and R. Roy, Phys. Rev. Lett. 60, 2626 (1988).

[4] W. Hohmann, J. Müller, and F. Schneider, J. Chem. Phys. 100, 5388 (1996).

[5] L. Gammaitoni et al., Rev. Mod. Phys. 70, 223 (1998).

[6] H. Gang et al., Phys. Rev. Lett. 71, 807 (1993).

[7] A. S. Pikovsky and J. Kurths, Phys. Rev. Lett. 78, 775 (1997).

[8] A. Neiman, P. I. Saparin, and L. Stone, Phys. Rev. E 56, 270 (1997).

[9] S. Fauve and F. Heslot, Phys. Lett. 97A, 5 (1983).

[10] G. Giacomelli et al., Phys. Rev. Lett. 84, 3298 (2000).

[11] K. Miyakawa and H. Isikawa, Phys. Rev. E 66, 046204 (2002).

[12] B. Lindner et al., Phys. Rep. 392, 321 (2004).

[13] I.Z. Kiss et al., Phys. Rev. E 67, 035201(R) (2003).

[14] O. V. Ushakov et al., Phys. Rev. Lett. 95, 123903 (2005).

[15] B. Lindner and L. Schimansky-Geier, Phys. Rev. E 61, 6103 (2000).

[16] C. Masoller, Phys. Rev. Lett. 88, 034102 (2002).

[17] C. Palenzuela et al., Europhys. Lett. 56, 347 (2001).

[18] L. S. Tsimring and A. Pikovsky, Phys. Rev. Lett. 87, 250602 (2001).

[19] L. Nunney, Theor. Popul. Biol. 27, 202 (1985).

[20] M. C. Mackey, J. Econ. Theory 48, 497 (1989).

[21] M. R. Roussel, J. Phys. Chem. 100, 8323 (1996).

[22] K. D. Choquette et al., Appl. Phys. Lett. 64, 2767 (1994).

[23] G. Giacomelli, F. Marin, and I. Rabbiosi, Phys. Rev. Lett. 82, 675 (1999).

[24] M. Sciamanna et al., Opt. Lett. 28, 1543 (2003).

[25] K. Panajotov et al., Phys. Rev. A 69, 011801(R) (2004).

[26] M. Arizaleta et al., Appl. Phys. Lett. 89, 091102 (2006).

[27] L. Gammaitoni et al., Phys. Rev. Lett. 62, 349 (1989).

[28] T. Zhou and F. Moss, Phys. Rev. A 41, 4255 (1990).

[29] T. Piwonski et al., Phys. Rev. Lett. 95, 040601 (2005).

[30] S. Barbay, G. Giacomelli, and F. Marin, Phys. Rev. E 61, 157 (2000).

[31] M. H. Choi, R. F. Fox, and P. Jung, Phys. Rev. E 57, 6335 (1998). 